\title{
Alteraciones del lenguaje en la esquizofrenia: Síntomas clínicos y medidas psicolingüísticas ${ }^{1}$
}

\section{RESUMEN}

Estudiamos la coherencia global del discurso en una muestra de sujetos con esquizofrenia y sujetos controles normales, encontrando diferencias significativas entre ambos que se mantienen en el tiempo

PALABRAS CLAVES: esquizofrenia, discurso, medidas psicolingüísticas.

\begin{abstract}
This paper presents the study of global coherence of discourse that shows a sample of schizophrenic patients and matched controls. We found differences statistically significatives within productions of both groups of subjects stables on time.
\end{abstract}

KEY WORDS: schizophrenia, discourse, psycholinguistic measures.

\section{1 - Introducción}

Distintos autores coinciden en que la evolución de los sujetos esquizofrénicos es heterogénea y la naturaleza de su pronóstico es multidimensional y se plantean dudas razonables a la hora de su delimitación y más aún, a la hora de referirnos a su evolución o a cómo predecirla (1-4).

La experiencia clínica nos sugiere, y queda avalado por múltiples trabajos (5-21), que son múltiples los factores y variables que intervienen en la evolución de la esquizofrenia; factores de tipo biológico, psicológico, socio-familiares (léase grupales), ambientales, etc. Igualmente las manifestaciones prodrómicas implican a los mismos factores (22-24).

Considerando la heterogeneidad referida, se hace necesario idear diversas estrategias para, sabiendo la dificultad que entraña, ir delimitando la evolución de los sujetos diagnosticados de esquizofrenia, con el fin de establecer programas de intervención. Por el mismo motivo, se hace necesario clasificar esta enfermedad mental en subtipos o fenotipos, con características de presentación (incluidas las alteraciones esquizofrénicas del lenguaje) y evolución similares.

Distintos autores proponen para la investigación en la esquizofrenia: a) la utilización de una metodología lo más precisa posible para la evaluación de su estado clínico (a nivel cualitativo y cuantitativo; tanto mediante el estudio de síntomas como de signos); b) la utilización conjunta de mediciones transversales y longitudinales (retrospectivas y prospectivas), para el estudio de su evolucióón (25); c) el estudio de 
diferentes dimensiones, a través de escalas no superpuestas de medición y que incluyan variables socio-bio-psicolágicas. Kay y Lindenmayer (26) señalan la necesidad de utilizar medidas no superpuestas en los estudios de predicción, al aumentar la posibilidad predictiva a medida que se establecen mediciones multidimensionales en una fase determinada de la enfermedad; e) la agrupación sindrómica en distintos subtipos lo más homogéneos posibles (27); y f) finalmente, habría un consenso en la comunidad científica de cara a que el reto en la actualidad pasa por la búsqueda de marcadores que identifiquen la "vulnerabilidad" para la esquizofrenia (individuos en riesgo), y que nos permitan prevenir nuevas recaídas (identificación de sujetos con pronóstico grave) (28-30).

\subsection{Concepto de vulnerabilidad a la esquizofrenia: signos vs. sintomas}

El concepto de "vulnerabilidad a la esquizofrenia" (31-33), se define como un "rasgo dimensional", duradero y relativamente permanente, frente al episodio esquizofréénico, que constituye un "estado" que sólo llega a producirse en ciertos individuos. Así, los marcadores de estado se relacionarían con el episodio de enfermedad (cuando ha aparecido) y los de rasgo detectarían riesgo genético y biológico (aún sin manifestarse).

Un marcador de rasgo tendría una serie de características: una distribución diferente en población psicótica/control; una estabilidad en el tiempo; una mayor prevalencia en miembros familiares; es capaz de predecir antes del desarrollo de las manifestaciones clínicas y es relativamente fiable y no invasivo.

Resultados obtenidos en investigaciones previas nos permitirían sostener, con las cautelas oportunas, que las alteraciones esquizofrénicas del lenguaje (medidas a través de índices psicolingüísticos) podrían cumplir buena parte de los requerimientos para poder ser consideradas marcadores de rasgo $(34,35)$.

Por lo tanto y en la línea de otros autores, (36-40), opinamos que la búsqueda de las claves para la explicación de esta grave enfermedad debe centrarse en el análisis de "signos" de funcionamiento que son constantes y objetivos en el sujeto y potencialmente previas al inicio de la enfermedad, y no tanto en los "síntomas clínicos" -subjetivosque sólo son observables durante el episodio psicótico o como residuo a lo largo de la enfermedad.

\subsection{El lenguaje como marcador}

Son varios los trabajos que estudian la especificidad del lenguaje esquizofrénico y su comportamiento durante una fase determinada de la enfermedad $(26,32,33,41-45)$, y menos los que investigan la evolución en el tiempo de las alteraciones esquizofrénicas del lenguaje $(46,47)$. Nuestra intención, ya desde estudios previos $(34,48)$ es el estudio de la evolución del lenguaje en tareas experimentales, a través de índices psicolingüísticos operativizables, para sopesar su capacidad como marcador de rasgo. 
Los trabajos citados anteriormente denominan y exploran las alteraciones del lenguaje observadas de diferentes maneras: a) como trastornos del pensamiento, lenguaje y comunicación (49-51), b) como pensamiento bizarro idiosincrásico $(43,47)$; c) como perfil psicótico (52); d) como comunicabilidad de los discursos (53); e) y otros -en nuestra línea- utilizando análisis psicolingüísticos, como déficit lingüísticos $(44,54-57)$. La pretensión es acceder al pensamiento y a lo que allí ocurre, a través de la conducta verbal observada, y estudiar así su relación con las características de la enfermedad (premóórbidas, de episodio y/o de evolución).

Los trabajos de Andreasen y su equipo, han sido de gran utilidad para confirmar y poner de manifiesto que las alteraciones del lenguaje de los sujetos con esquizofrenia son alteraciones extraordinariamente heterogéneas y variables, y han permitido iniciar el estudio operativo de las alteraciones del lenguaje en la esquizofrenia desde una vertiente clínica, marcando una pauta de inicio para dicha operativización $(46,49,50,58-61)$.

Por otro lado los trabajos de Harrow y sus colaboradores $(43,47,62,63)$ estudian el lenguaje no sólo a través de sus manifestaciones clínicas, sino a través de tareas verbales específicas y replicables, intentando buscar e identificar patrones básicos de respuesta y marcando el inicio del estudio del perfil del habla en la esquizofrenia a través de pruebas experimentales.

\subsection{Análisis psicolingüisticos de las alteraciones del lenguaje en la esquizofrenia.}

Desde los años 60, se ha realizado un amplio grupo de trabajos experimentales que, en lugar de utilizar juicios clínicos, parten de medidas derivadas directamente de los modelos psicolingüísticos para el estudio del lenguaje normal (es decir, utilizan "signos" y no sólo "síntomas" en la investigación científica del trastorno).

Los estudios realizados desde esta perspectiva -analizados en profundidad y replicados por Belinchón (64-67)- muestran, cuando menos, dos resultados dignos de mención.

Por un lado, el dato (confirmado con una gran variedad de tareas), de que los sujetos con esquizofrenia utilizan por lo general el lenguaje de una forma adecuada aunque (ocasionalmente y con una probabilidad significativamente más alta que sus controles normales), no lo hacen de una forma práctica, esto es, construyen mensajes que no cubren adecuadamente las necesidades comunicativas de sus interlocutores (incluyen referentes ambiguos o implícitos que deberíáan ser explícitos, no respetan el orden lógico de presentación de la información requerido por los oyentes, están menos cohesionados de lo necesario, etc.). Así, podemos decir que los sujetos con esquizofrenia hacen un mal uso de las reglas lingüísticas de la codificación textual, produciendo textos anómalos justo en el tipo de indicadores lingüísticos que hacen referencia a la competencia pragmática. 
En segundo lugar, algunos trabajos han puesto de manifiesto la posibilidad de que los sujetos con esquizofrenia sufran alteraciones estructurales específicas (morfosintácticas y textuales) además de las ya referidas alteraciones de planificación comunicativa.

Este trabajo, y en el marco más amplio de un estudio en que estamos evaluando las alteraciones de otros componentes del discurso en la esquizofrenia, intenta operacionalizar la macroestructura o coherencia global del discurso que muestran los sujetos en sus producciones verbales, en este caso, comparando los discursos producidos en dos momentos distintos (tiempo 0 y 9 meses más tarde) por sujetos con esquizofrenia con los discursos producidos por sujetos controles normales, a través del análisis formal del discurso grabado en una situación estructurada, siendo la tarea requerida a los sujetos el contar una historia previamente narrada por el interlocutor.

\section{2.- MÉTODO}

\subsection{Selección de la muestra}

La muestra estaba compuesta por 10 sujetos con esquizofrenia y 11 sujetos controles normales, entendiendo por normales la ausencia de psicopatología y de síntomas psiquiátricos.

Los sujetos con esquizofrenia fueron seleccionados por conveniencia ${ }^{2}(68)$ en base a una serie de criterios: diagnóstico clínico DSM-IV, edad entre 18 y 35 años, duración previa de la enfermedad de más de dos años (para asegurar su diagnóstico definitivo de esquizofrenia), no estar en un momento de exacerbación aguda de la enfermedad (ni estado de descompensación psicótica ni estado de depresión post-psicótica) y encontrarse en tratamiento ambulatorio regulado, estabilizado y con una red social de apoyo en el momento del estudio (todos los sujetos vivían con sus padres y/o su pareja quienes participaban activamente en el tratamiento del familiar con esquizofrenia, quien acudía a un Hospital de Día de la red de Salud Mental extrahospitalaria). Asimismo ninguno cumplía los criterios DSM-IV para los "Trastornos relacionados con sustancias", y todos tenían una puntuación en la escala verbal del WAIS $>90$.

Los sujetos controles se seleccionaron intentando homogeneizar las características sociodemográficas, prestando especial atención a la edad, lugar de residencia, nivel socioeconómico y de estudios obtenido y a la puntuación verbal del WAIS.

La participación de los sujetos en el estudio fue completamente voluntaria y consentida.

La pasación de todas las escalas, así como la narración y grabación del protocolo de lenguaje, se realizó en formato de entrevista estructurada y cada entrevista tuvo una

2 Las muestras por conveniencia se escogen en base a la oportunidad y accesibilidad de los sujetos, favorecienco la posibilidad de trabajar con grupos inaccesibles en muchas circunstancias, en detrimento de las posibilidades de generalización de los resultados. 
duración de aproximadamente 1 hora y media. Todas las pruebas se administraron al comienzo del estudio (T0) y en un segundo momento, 9 meses más tarde (T1).

\subsection{Evaluación clínica y sintomatológica}

Se utilizaron las escalas para la evaluación de los síntomas positivos (SAPS) y de síntomas negativos (SANS) de Andreasen $(60,61)$, por ser los instrumentos de referencia para la evaluación clínica de la esquizofrenia y contar con una versión en español (69) y varios estudios de validación (70-72). Asimismo utilizamos la versión en español de la escala T.L.C. (Thought, Language and Communication) para la evaluación clínica del lenguaje (73-75).

Para la medición del funcionamiento global, se utilizó la Escala de Evaluación Global (GAS ó EEAG) de Endicott y cols. (76); la Escala de Impresión Clínica Global (CGI) (77); la Escala de Evolución de Strauss y Carpenter (78) y la escala de Calidad de Vida de Ruiz y Baca (79).

Para la valoración del ajuste premórbido, se utilizó la escala Premorbid Adjustment Scale (PAS) (80); y para la valoración de la psicopatología, utilizamos la Brief Psychiatric Rating Scale (B.P.R.S.) (81) para valorar a los sujetos con esquizofrenia y a los controles normales; y la escala SCL-90 (82) autoaplicada para valorar la normalidad en los sujetos controles normales.

\subsection{Evaluación psicolingüistica}

Siguiendo la línea de estudios previos $(34,64)$, se seleccionó una tarea de producción verbal, consistente en la narración de una historia previamente presentada por el entrevistador (ver Anexo I), y el análisis de aquellos índices psicolingüísticos que operativizan la organización semántica global del discurso (macroestructura textual), dimensión que hace referencia a la coherencia global del texto, asumiendo una definición compleja de la noción teórica de "coherencia" $(64,83)$.

Esta dimensión se evalúa a partir de cuatro parámetros que se operativizan en diferentes índices y que se describen a continuación.

a) Calidad representacional de los constituyentes textuales: depende de que se incluyan o no en la narración que realiza el sujeto las unidades semánticas que son básicas para la (re) construcción del guión o esquema argumental de la historia que se ha presentado previamente.

Los 4 índices que operativizan la calidad representacional de los constituyentes textuales son:

1) Porcentaje de Módulos completos sobre el total de módulos de la narración: Los módulos completos incluyen la información argumentalmente básica y al menos parte de la información secundaria que corresponde a tal módulo. 
2) Porcentaje de Módulos incompletos sobre el total de módulos de la narración: Los módulos incompletos sólo contienen el elemento nuclear de la información

3) Porcentaje de Módulos insuficientes sobre el total de módulos de la narración: Los módulos insuficientes sólo contienen información argumentalmente necesaria.

4) Porcentaje de Módulos que contienen Distorsiones y Adiciones sobre el total de módulos de la narración: Las Distorsiones y Adiciones son errores o información argumentalmente irrelevante que ha sido incluida en determinados módulos.

b) Secuenciación u ordenación temporal correcta de los hechos o eventos narrados: La ordenación temporal se operativiza a través de dos índices:

1) El porcentaje de módulos con errores de orden intra-constituyentes: errores que afectan a la ordenación de los eventos dentro del propio constituyente

2) El porcentaje de módulos con errores de orden inter-constituyentes: errores que afectan a la ubicación del constituyente en la secuencia total del discurso

c) Conexión entre los constituyentes textuales del discurso, operativizada a través de 3 índices:

1) Porcentaje de conexiones adecuadas y explícitas entre los módulos sobre el total de conexiones: en ellas, el hablante vincula temática o temporalmente el contenido de los constituyentes.

2) Porcentaje de conexiones adecuadas e implícitas entre los módulos sobre el total de conexiones: en ellas, el hablante superpone los constituyentes de forma correcta pero sin vincularlos de forma explícita.

3) Porcentaje de conexiones inadecuadas entre los módulos sobre el total de conexiones: en ellas, el hablante distorsiona las relaciones de conexión y/o el orden de los constituyentes del texto.

d) Congruencia con el texto original (esta es una dimensión que sólo es aplicable a narraciones sobre eventos previamente conocidos por el examinador y del que se conoce su estructura informativa completa). Esta dimensióón se operacionaliza a través de 1 índice:

1) porcentaje de constituyentes textuales incluidos en la (re)construcción de la narración sobre el total de constituyentes que contiene el texto original.

Segmentamos el texto producido por los sujetos experimentales y controles en los constituyentes textuales o módulos, a partir del listado de episodios de que consta el 
material original utilizado (los episodios principales de la "Historia del pirata Landolfo" adaptados por Belinchón (64) sobre Van Dijk (84)).

Para el análisis estadístico de los datos, hemos realizado primero un análisis exploratorio (EDA) y posteriormente descriptivos, análisis de correlación, comparación de las puntuaciones entre ambos grupos de sujetos mediante la utilización de pruebas no paramétricas (teniendo en cuenta el tamaño de la muestra y la dificultad para el cumplimiento de condiciones de normalidad y homocedasticidad): la prueba de rangos con signo de Wilcoxon (para las muestras relacionadas) y la prueba de MannWhitney (para muestras independientes). Se utilizó el paquete estadístico SPSS 9.0 para Windows.

\section{3.- RESULTADOS}

\subsection{Descripción de la muestra}

En la Tabla 1 se recogen las características sociodemográficas de los sujetos de ambos grupos.

\subsection{Características clínicas de la muestra}

En la Tabla 2, presentamos las características clínicas de la muestra de sujetos con esquizofrenia, medidas tanto en el tiempo 0 (T0) como en el primer seguimiento (T1).

Desde el punto de vista sintomatológico, se observan diferencia significativas en el tiempo en los indicadores de síntomas negativos (SANS Puntuación Total T0: Media= 10.4; dt $=2.6$ y SANS Puntuación Total T1: Media $=11.9 ; \mathrm{dt}=3.87 ; \mathrm{z}=1.98 ; \mathrm{p}$ $<.05$; así como SANS Puntuación Compuesta T0: Media $=50 ; \mathrm{dt}=13.9$ y SANS Puntuación Compuesta T1: Media $=58.6 ; \mathrm{dt}=18 ; \mathrm{z}=2.45 ; \mathrm{p}<.05)$ y en el subfactor "Activismo" (T0: Media $=2.4 ; \mathrm{dt}=0.77 ; \mathrm{T} 1$ : Media $=2.97 ; \mathrm{dt}=1.07 ; \mathrm{z}=1.97 ; \mathrm{p}$ $<.05)$ de la escala B.P.R.S.

El comportamiento de la escala T.L.C. no sufre variaciones significativas a lo largo del tiempo (T.L.C. T0: Media $=21.6 ; \mathrm{dt}=3.9$; T.L.C. T1: Media $=23.8 ; \mathrm{dt}$ $=7.23 ; \mathrm{z}=1.19, \mathrm{p}<.25)$.

Tampoco encontramos variaciones en el tiempo en la escala EEAG $(z=.46$; $\mathrm{p}<.65)$; en la escala de evolución $(\mathrm{z}=.95 ; \mathrm{p}<.35)$, en las puntuaciones de la escala de Calidad de Vida $(\mathrm{z}=.96 ; \mathrm{p}<.35)$, ni en las puntuaciones de la autovaloración del estado de salud $(\mathrm{z}=1.43 ; \mathrm{p}<.16)$.

Por otro lado, y como se aprecia en la Tabla 3; la muestra de sujetos controles normales presenta unas características también estables en el tiempo, sin observarse cambios significativos al comparar las mediciones en los tiempos 0 y 1 , excepto en las 
subescalas Ansiedad-Depresión, Trastornos del Pensamiento y Activismo del B.P.R.S. que aunque varían lo hacen en dirección hacia la normalidad. Es decir, en el T1 siguen comportándose como sujetos controles normales.

Obviamente, se encuentran perfiles distintos (con diferencias estadísticamente significativas) en todas las subescalas de la escala B.P.R.S. entre los sujetos con esquizofrenia y la muestra de controles normales.

A la hora de pasar la escala T.L.C. a los sujetos controles normales, nos hemos encontrado (como era de esperar) con puntuaciones excesivamente bajas.

\subsection{Características de las alteraciones del lenguaje en la macroestructura del texto}

El análisis de correlaciones muestra una alta correlación entre los índices psicolingüísticos que operacionalizan la macroestructura del texto entre sí, tanto en el grupo de sujetos controles como en el grupo de sujetos con esquizofrenia en ambos tiempos (T0 y T1), revelando que éstos están midiendo diferentes aspectos de una misma dimensión.

Las Tablas 5 y 6 presentan los datos de la comparación de los índices psicolingüísticos que operacionalizan la macroestructura o coherencia global de los discursos, entre los sujetos con esquizofrenia y los sujetos controles normales tanto en el T0 como en el T1.

Como se puede observar, todos los índices psicolingüísticos muestran diferencias estadísticamente significativas en la comparación de ambos grupos, con la excepción de "módulos incompletos" y "conexiones adecuadas e implícitas" en el T0, y "módulos incompletos" en el T1. Encontramos una diferencia tendencial para el índice "número de constituyentes textuales" en el T0.

A su vez, las Tablas 7 y 8 presentan la comparación de los índices psicolingüísticos que operacionalizan la macroestructura del texto en ambos momentos (T0 y T1) tanto para los sujetos con esquizofrenia como para los controles normales.

Como puede observarse, ninguno de los índices psicolingüísticos (excepto uno) muestra diferencias estadísticamente significativas en la comparación de ambos tiempos para el grupo de sujetos con esquizofrenia, señalando que el patrón de coherencia global del texto es el mismo en T0 y T1. Sólo aumentan las "conexiones adecuadas e implícitas" en T1, aunque siempre este índice es menor para los sujetos con esquizofrenia que para los controles normales.

Asimismo, también en la muestra de sujetos controles se mantiene el patrón macroestructural ya que sólo dos índices muestran diferencias en el tiempo. Los sujetos 
controles en T1 realizan menos "conexiones adecuadas y explícitas" y más "conexiones adecuadas e implícitas" que en T0, aunque siempre significativamente más que los sujetos con esquizofrenia.

\section{4.- DISCUSIÓN}

Como se observa en los resultados presentados, encontramos desde el punto de vista de la evolución clínica y dentro de la estabilidad de la muestra de sujetos con esquizofrenia, una ligera variación en la dirección de la negativización (incluyendo itemes como bloqueo afectivo, entorpecimiento motor, repliegue emocional, manierismos, estereotipias, etc.), confirmando los resultados de estudios previos que señalan una negativización de las muestras a lo largo del tiempo.

En la valoración del lenguaje desde el punto de vista clínico (operacionalizado por la puntuación en la escala T.L.C.), encontramos escasas variaciones a lo largo del tiempo, con puntuaciones similares al subtipo mixto de nuestros estudios previos (media $=21.45 \mathrm{y} \mathrm{dt}=5.8$ ) (34) y en relación con las escalas SANS y SAPS. El que no haya mostrado una significativa diferencia en la evolución (a diferencia del SANS) y dado que en los estudios citados realizados se ha comportado de forma errática, puede indicar una menor sensibilidad de la escala T.L.C. en períodos de no reagudización que la escala de sintomatología negativa.

A la hora de pasar la escala T.L.C. a los sujetos controles normales, nos hemos encontrado con puntuaciones excesivamente bajas, lo que cabría esperar a partir de la inexistencia de puntuaciones en las escalas SANS y SAPS (que incluyen parcialmente la escala T.L.C.) para esta muestra control.

Esta situación refuerza el interés que tiene estudiar índices del lenguaje que se presenten tanto en sujetos normales como en sujetos psiquiátricos y que posibiliten el análisis y el tratamiento estadístico de los datos del discurso en distintas poblaciones.

Por otro lado, todos los índices psicolingüísticos operacionalizados para medir la macroestructura textual, excepto uno, muestran diferencias significativas al comparar ambos grupos de sujetos. Así, los índices que operacionalizan la calidad representacional o informativa de los constituyentes textuales, los que operativizan la secuenciación $u$ ordenación temporal correcta de los hechos narrados; los que dan cuenta de las conexiones entre los constituyentes textuales y el que operacionaliza la congruencia del discurso producido con el texto original; diferencian significativamente el discurso de los sujetos con esquizofrenia del discurso de los sujetos controles normales.

Sólo en un índice ("módulos incompletos") obtenemos casi las mismas puntuaciones para sujetos con esquizofrenia que para sujetos controles, no siendo significativa la diferencia entre ambos grupos. Este índice es uno de los que operativiza 
la dimensión "calidad representacional de los constituyentes textuales" y hace referencia a que en la narración de la historia, y para un determinado módulo, se incluya sólo el elemento nuclear de la información, obviando información secundaria que está presente.

Por otro lado, cuando comparamos a los sujetos consigo mismos en el tiempo, observamos que la macroestructura del discurso se mantiene estable. Los sujetos con esquizofrenia realizan más "conexiones adecuadas e implícitas" en el T1, pero siempre menos que los controles normales. Por su parte, estos sujetos normales realizan menos “conexiones adecuadas y explícitas" y más "conexiones adecuadas e implícitas" en el T1. La diferencia entre ambos índices es que en uno se explicita la vinculación temática y en el otro no, aunque ambas son conexiones adecuadas. El resto de los índices utilizados, evidencia diferencias claras en los discursos de ambos grupos de sujetos.

Así, a nuestro entender y siendo cautos en nuestras afirmaciones -teniendo en cuenta el número de sujetos-, el estudio la organización macroestructural del discurso producido por sujetos con y sin esquizofrenia a través de índices psicolingüísticos, muestra dos cuestiones a señalar:

a) En primer lugar, encontramos diferencias estadísticamente significativas en los índices que operativizan la coherencia global del discurso entre los sujetos con esquizofrenia y los controles normales, en el sentido de que los mismos índices estarían presentes en ambas poblaciones pero con una distribución distinta según de qué índices hablemos.

Así, el dar información suficiente (nuclear y secundaria) de un suceso y conectar adecuada y explícitamente los eventos unos con otros estaría en relacióón con los sujetos normales; y el no presentar información suficiente para la comprensión del discurso, presentar distorsiones e información adicional, errores dentro de un mismo constituyente en el orden de los hechos y conexiones inadecuadas entre los grupos de eventos, estaría en relación con los sujetos con esquizofrenia.

La diferencia en la estructuración y ordenación de los constituyentes macrotextuales del discurso encontrada entre ambos grupos de sujetos, es coherente con las hipótesis sostenidas en otros estudios acerca de la existencia de un déficit cognitivo general en los sujetos con esquizofrenia, ya que si analizamos los procesos que funcionalmente configuran la primera fase de la producción verbal, vemos son procesos difícilmente automatizables; tratándose, por tanto de procesos controlados que requieren una elevada demanda atencional $(85,86)$. Se hace indispensable, pues, el control de la capacidad para mantener la atención en una tarea, tanto de los sujetos con esquizofrenia como de los sujetos controles, y estudiar su relación con los discursos producidos.

No obstante, y como señala Belinchón (67), los sujetos con esquizofrenia podrían tener alterados los "componentes autónomos del procesamiento lingüístico" 
(componentes que operan en la fase de codificación lingüística); y, además de los déficit observados en la codificación pragmática y macroestructural de los discursos, sufrir perturbaciones de naturaleza morfosintáctica. Estudios diseññados específicamente para la investigación de esta fase del procesamiento, contribuirán al esclarecimiento de estas cuestiones.

b) En segundo lugar, encontramos una estabilidad, de los índices, de sus valores y de la diferencia entre sujetos normales y sujetos con esquizofrenia a lo largo del tiempo

Esta estabilidad en el tiempo que hemos encontrado, nos alienta para seguir trabajando de cara a intentar encontrar componentes del lenguaje que pudieran servir como marcador de rasgo o marcador estable en la esquizofrenia; ya que a pesar de la cantidad de estudios transversales que señalan diferencias en distintos procesos del procesamiento de la información entre los sujetos con esquizofrenia y los sujetos controles, en pocas ocasiones se ha puesto de manifiesto el carácter episódico o estable del indicador o supuesto marcador utilizado. En esta línea, continuamos el seguimiento de los sujetos de nuestra muestra y nos planteamos la necesidad de estudiar a sujetos en distintos momentos de la enfermedad, así como a sus familiares directos.

Así, creemos necesario que continuar con el estudio experimental operativo de índices psicolingüísticos en poblaciones psiquiátricas y no psiquiátricas, antes, durante y después del inicio de la enfermedad; ya que consideramos que estos estudios tienen un valor teórico y heurístico; es decir, tanto para la práctica clínica como para la investigación, y que nos pueden ayudar a ser cada vez más rigurosos en la delimitación, valoración y operativización de determinadas características del lenguaje en las distintas psicopatologías.

\section{ANEXO:}

Versión de la "Historia del corsario Landolfo Rufolo", de Bocaccio

"En un hermoso lugar de las costas de Italia, hace ya algunos años, vivía un rico comerciante llamado Landolfo. Landolfo era dueño de inmensas riquezas pero su codicia era tan grande que vivía obsesionado con la idea de doblar su fortuna. Con el fin de hacer un buen negocio, compró un barco muy grande que cargó con mercancías diversas, y zarpó con él hacia Chipre. Pero al llegar allí descubrió que otros muchos barcos habían fondeado antes que él vendiendo el mismo tipo de mercancías que las que él había llevado consigo. Por esta razón, se vio obligado a vender sus mercancías a precio de liquidación y quedó practicamente arruinado.

Como no quería regresar a su patria arruinado, decidió convertirse en pirata para recuperar el dinero perdido. Vendió su buque y con el dinero compró un barco ligero. Lo equipó adecuadamente y recorrió los mares saqueando todos los barcos que 
pudo. Tuvo tanto éxito en su nuevo oficio que en poco más de un año recuperó las pérdidas de sus negocios de comerciante y obtuvo una fortuna mucho mayor que la que poseía antes.

Considerando que ya tenía bastante dinero, decidió regresar a su patria. Pero apenas iniciado el viaje de vuelta, se levantó una tormenta tan fuerte que Landolfo se vio obligado a refugiarse en una pequeña isla. Poco después llegaron a la misma isla unos comerciantes genoveses. Al reconocer al pirata Landolfo, decidieron atacarle y hacerlo su prisionero.

Al día siguiente, los comerciantes genoveses se echaron a la mar llevando a Landolfo encerrado en la bodega de uno de sus barcos. Pero de nuevo se levantó una fuerte tormenta. Los barcos fueron arrojados contra las rocas y se hicieron pedazos. Landolfo, para salvarse, se encaramó a una caja, se tendió sobre ella y, sirviéndose de los brazos para guiarla logró mantenerse a flote.

Pasó un día y una noche a la deriva, sin probar bocado y sin saber donde se encontraba. Al fin empujado por la propia violencia del viento y convertido ya casi en una esponja, Landolfo llegó a la costa de la isla de Corfú. En aquel momento, en la playa, una mujer estaba fregando con arena sus utensilios de cocina. Al verlo, la mujer se asustó, pero viéndole tan agotado, decidió ayudarle. Se acercó a él y lo llevó a su casa. Allí le dió masajes, un baño caliente, comidas y bebidas que lo reconfortaron. Cuando estuvo ya completamente restablecido, la mujer le devolvió la caja que le había salvado del naufragio.

Un día, mientras la mujer estaba ausente de la casa, Landolfo abrió la caja. Descubrió que estaba llena de joyas valiosas y que podía hacerse rico con su venta. Dio gracias a Dios por su suerte y decidió regresar a su patria. Allí vendió las joyas. Con el dinero que obtuvo por ellas consiguió una fortuna que era el doble de grande que la que tenía al principio. Envió una importante suma de dinero a la mujer de Corfú que le había sacado del mar, como pago por su ayuda. Nunca más se interesó por el comercio : dedicó su dinero a vivir con todo esplendor y tranquilidad el resto de sus días" 
TABLA 1: DATOS SOCIODEMOGRÁFICOS DE LOS SUJETOS

\begin{tabular}{|c|c|c|c|}
\hline & & $\begin{array}{l}\text { Esquizofrenta } \\
(n=10)\end{array}$ & $\begin{array}{l}\text { Controles } \\
\text { Controles }(n=11)\end{array}$ \\
\hline Edad & $\begin{array}{l}\text { Media } \\
\text { Desviación típica }\end{array}$ & \begin{tabular}{|l|}
31.5 \\
3.06 (Rango: 26-35) \\
\end{tabular} & \begin{tabular}{|l}
27.5 \\
4.63 (Rango: 24-38) \\
\end{tabular} \\
\hline Sexo & \begin{tabular}{|l} 
Mujeres \\
Hombres
\end{tabular} & $\begin{array}{l}6(60 \%) \\
4(40 \%)\end{array}$ & $\begin{array}{l}4(36.4 \%) \\
7(63.6 \%)\end{array}$ \\
\hline $\begin{array}{l}\text { Lugar de } \\
\text { residencia }\end{array}$ & Urbano (población $<84.000$ ) & $10(100 \%)$ & $11(100 \%)$ \\
\hline $\begin{array}{l}\text { Situación en } \\
\text { el domicilio }\end{array}$ & $\begin{array}{l}\text { Hijo/a: } \\
\text { Pareja: }\end{array}$ & \begin{tabular}{|l}
$7(70 \%)$ \\
$3(30 \%)$ \\
\end{tabular} & $\begin{array}{l}8(72.7 \%) \\
3(27.3 \%) \\
\end{array}$ \\
\hline Nivel educativo & $\begin{array}{l}\text { ESO } \\
\text { Bachillerato superior } \\
\text { Menos de } 7 \text { años de escol. } \\
\text { Bachillerato inacabado } \\
\text { Diplomatura } \\
\text { Licenciatura } \\
\end{array}$ & $\begin{array}{l}5(50 \%) \\
2(20 \%) \\
1(10 \%) \\
1(10 \%) \\
- \\
1(10 \%) \\
\end{array}$ & $\begin{array}{l}2(18.2 \%) \\
5(54.5 \%) \\
- \\
2(18.2 \%) \\
2(18.2 \%) \\
- \\
\end{array}$ \\
\hline Situación laboral & \begin{tabular}{|l} 
Parado \\
Pensionista \\
Ama de casa \\
Trabajador retribuido \\
Estudiante \\
\end{tabular} & \begin{tabular}{|l|}
$5(50 \%)$ \\
$2(20 \%)$ \\
$2(20 \%)$ \\
$1(10 \%)$ \\
- \\
\end{tabular} & $\begin{array}{l}- \\
- \\
2(18.2 \%) \\
8(72.7 \%) \\
1(9.1 \%) \\
\end{array}$ \\
\hline Estado civil & \begin{tabular}{|l|} 
Soltero \\
Casado \\
Vive en pareja \\
\end{tabular} & $\begin{array}{l}7(70 \%) \\
3(30 \%) \\
-\end{array}$ & \begin{tabular}{|lc}
$9(81.8 \%)$ \\
$1(9.1 \%)$ \\
$1(9.1 \%)$ \\
\end{tabular} \\
\hline Diagnóstico & $\begin{array}{l}\text { Esquizofrenia paranoide } \\
\text { Esquizofrenia desorganizada } \\
\text { Esquizofrenia indiferenciada }\end{array}$ & $\begin{array}{l}7(70 \%) \\
2(20 \%) \\
1(10 \%) \\
\end{array}$ & \\
\hline Tratamiento farm & acológico & $10(100 \%)$ & \\
\hline Cumplimentación & \begin{tabular}{|l} 
Buena \\
Regular
\end{tabular} & \begin{tabular}{|l|}
$8(80 \%)$ \\
$2(20 \%)$
\end{tabular} & \\
\hline \multicolumn{2}{|c|}{ Media de ingresos psiquiátricos } & 1.7 (Rango: 0-3) & \\
\hline \multicolumn{2}{|c|}{ Media de dras ingresado } & 50.6 (Rango: 0-106) & \\
\hline \multicolumn{2}{|c|}{ Media de edad alprimer ingreso } & 25.4 (Rango: 18-32) & \\
\hline \multicolumn{2}{|c|}{ Antecedentes psiquiátricos familiares } & $6(60 \%)$ & \\
\hline
\end{tabular}


TABLA N ${ }^{\circ}$ 2: DESCRIPCIÓN CLÍNICA DE LA MUESTRA DE SUJETOS CON ESQUIZOFRENIA (T0 y T1)

\begin{tabular}{|c|c|c|c|c|c|}
\hline & iv & Medih & alt & $\frac{1}{4}$ & Sigr (bingt) \\
\hline 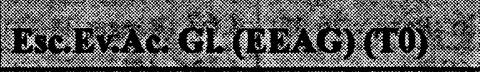 & 10 & 46.3 & 13.4 & \multirow[t]{2}{*}{.46} & \multirow[t]{2}{*}{.646} \\
\hline 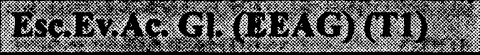 & 10 & 43.9 & 10.7 & & \\
\hline 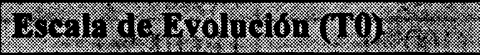 & 10 & 2.9 & .77 & \multirow[t]{2}{*}{.95} & \multirow[t]{2}{*}{.341} \\
\hline 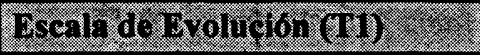 & 10 & 2.63 & .78 & & \\
\hline Caliaed deVida (ro) & 10 & 3.6 & .66 & \multirow[t]{2}{*}{1.47} & \multirow[t]{2}{*}{.141} \\
\hline Calidgd de Vida (T1) & 10 & 3.18 & .63 & & \\
\hline Antorkin Estado Salud $(\mathrm{TO})^{2}$ & 10 & 72 & 18.1 & \multirow[t]{2}{*}{1.43} & \multirow[t]{2}{*}{.152} \\
\hline Autoval. Estado Salud (TI) & 10 & 57 & 21.3 & & \\
\hline SANS $(\mathrm{P} \cdot \operatorname{toth})$ (T0) & 10 & 10.4 & 2.6 & \multirow[t]{2}{*}{1.98} & \multirow[t]{2}{*}{.048} \\
\hline SAINS $(\mathrm{f}$ totan $(\mathrm{CD})$ & 10 & 11.9 & 3.87 & & \\
\hline SAINS (P. compiesta) (Tro) & 10 & 50 & 13.9 & \multirow[t]{2}{*}{2.45} & \multirow[t]{2}{*}{.014} \\
\hline SANS $(\mathrm{P}$. compuesta) $(\mathrm{T} 1)$ & 10 & 58.6 & 18 & & \\
\hline SAPS (P. total) (TO) & 10 & 9.1 & 2.6 & \multirow[t]{2}{*}{.49} & \multirow[t]{2}{*}{.621} \\
\hline SAPS (F total) (11) & 10 & 8.8 & 3.1 & & \\
\hline SAPS (P. computesta) (T0) & 10 & 62.7 & 18.4 & \multirow[t]{2}{*}{.35} & \multirow[t]{2}{*}{.720} \\
\hline SAPS $(\mathrm{F}$ compuesta) $(T 1)$ & 10 & 60.5 & 23.8 & & \\
\hline \multicolumn{6}{|l|}{ B.PRSE (sublactortes) } \\
\hline Ansiedad-Deprcrión (TO) & 10 & 2.7 & .91 & \multirow[t]{2}{*}{.29} & \multirow[t]{2}{*}{.766} \\
\hline Ansiedad-Deprosion (II) & 10 & 2.8 & .75 & & \\
\hline Anergia (TO) & 10 & 2.5 & .93 & \multirow[t]{2}{*}{1.89} & \multirow[t]{2}{*}{.058} \\
\hline 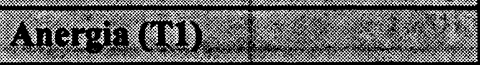 & 10 & 2.95 & .77 & & \\
\hline Trastornos Pencamiento (TO) & 10 & 2.4 & .88 & \multirow[t]{2}{*}{1.12} & \multirow[t]{2}{*}{.260} \\
\hline Trastornos Ponsimiento (II) & 10 & 2.07 & .82 & & \\
\hline Activismo $(\mathrm{T} 0)$ - & 10 & 2.4 & .77 & \multirow[t]{2}{*}{1.97} & \multirow[t]{2}{*}{.049} \\
\hline 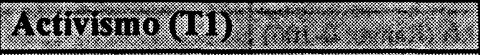 & 10 & 2.97 & 1.07 & & \\
\hline Hostiliand-Suspicacia (TO) & 10 & 2.1 & .72 & \multirow[t]{2}{*}{1.41} & \multirow[t]{2}{*}{.156} \\
\hline Hostilidad-Suspicacia (T1) & 10 & 2.77 & 1.23 & & \\
\hline
\end{tabular}


TABLA N ${ }^{\circ}$ 3: DESCRIPCIÓN CLÍNICA DE LA MUESTRA DE SUJETOS CONTROLES NORMALES (T0 y T1)

\begin{tabular}{|c|c|c|c|c|c|}
\hline & $\mathrm{N}$ & Media & dt: & $(-2)$ & Stge(bilat) \\
\hline BRRS. (cubractores) & & & & & \\
\hline Ansiedad-Depresion $(\mathrm{TO})$ & 11 & 1.82 & .23 & \multirow[t]{2}{*}{2.83} & \multirow[t]{2}{*}{.005} \\
\hline 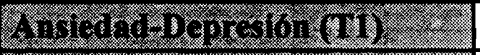 & 11 & 1.41 & .17 & & \\
\hline Arorgit $(10)=0$ & 11 & 1.14 & .21 & \multirow[t]{2}{*}{.276} & \multirow[t]{2}{*}{.783} \\
\hline 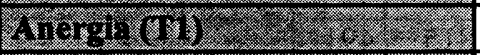 & 11 & 1.11 & .17 & & \\
\hline Thastornos Ponsunfento (TO) & 11 & 1.25 & .19 & \multirow[t]{2}{*}{2.6} & \multirow[t]{2}{*}{.009} \\
\hline Ther onios Peibamiento (T1) & 11 & 1.00 & .00 & & \\
\hline Activisulo (10) & 11 & 1.39 & .33 & \multirow[t]{2}{*}{2.12} & \multirow[t]{2}{*}{.033} \\
\hline Activismo (II) & 11 & 1.15 & .27 & & \\
\hline 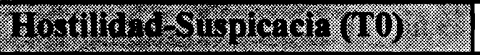 & 11 & 1.36 & .23 & \multirow[t]{2}{*}{.355} & \multirow[t]{2}{*}{.723} \\
\hline Iostillo d Suguchora (T) & 11 & 1.45 & .52 & & \\
\hline \multicolumn{6}{|l|}{ Scl-90 (cub hotores) } \\
\hline Somintizacion(TO) : & 11 & .41 & .23 & \multirow[t]{2}{*}{1.6} & \multirow[t]{2}{*}{.108} \\
\hline Somhthactom (II) & 11 & .58 & .33 & & \\
\hline Obsesion-Compusion (10) & 11 & .75 & .40 & \multirow[t]{2}{*}{1.39} & \multirow[t]{2}{*}{.164} \\
\hline Obsesion Compulsion (II) & 11 & .97 & .66 & & \\
\hline Iflpe senvibllogd (TO) $=8$ & 11 & .98 & .51 & \multirow[t]{2}{*}{.46} & \multirow[t]{2}{*}{.645} \\
\hline Hibersensibilldad (T) & 11 & .89 & .39 & & \\
\hline 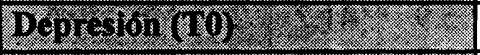 & 11 & .62 & .35 & \multirow[t]{2}{*}{1.29} & \multirow[t]{2}{*}{.195} \\
\hline Depresibit (TI) : & 11 & .79 & .45 & & \\
\hline Anvichat $(10)$ - & 11 & .46 & .32 & \multirow[t]{2}{*}{.102} & \multirow[t]{2}{*}{.919} \\
\hline 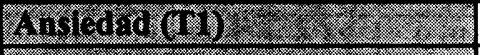 & 11 & .45 & .41 & & \\
\hline Hostifdad $(00)-30$ & 11 & .97 & .66 & \multirow[t]{2}{*}{1.07} & \multirow[t]{2}{*}{.283} \\
\hline 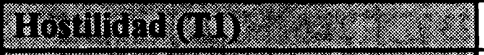 & 11 & .80 & .71 & & \\
\hline Anstedad robles (TO) & 11 & .07 & .09 & \multirow[t]{2}{*}{1.72} & \multirow[t]{2}{*}{.084} \\
\hline 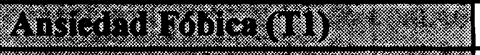 & 11 & .19 & .24 & & \\
\hline Permolnsmo (10) & 11 & .98 & .69 & \multirow[t]{2}{*}{.154} & \multirow[t]{2}{*}{.878} \\
\hline Paramoldkmo (TI) & 11 & .94 & .56 & & \\
\hline 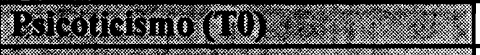 & 11 & .49 & .47 & \multirow[t]{2}{*}{.256} & \multirow[t]{2}{*}{.798} \\
\hline Psicotioismo $(0)]=0.0$ & 11 & .43 & .31 & & \\
\hline Escele A d & 11 & .65 & .44 & \multirow[t]{2}{*}{.07} & .944 \\
\hline Escaln Adiclongl (TI) & 11 & .57 & .34 & & \\
\hline
\end{tabular}


TABLA N ${ }^{\circ}$ 4: CORRELACIONES ENTRE LOS ÍND. PSICOLINGUÍSTICOS

\begin{tabular}{|c|c|c|c|c|c|c|c|c|c|}
\hline & $\begin{array}{l}\text { Modulios } \\
\text { Coninplet. }\end{array}$ & $\begin{array}{l}\text { Modul. } \\
\text { Incoupp. }\end{array}$ & $\begin{array}{l}\text { Modul } \\
\text { Insuric: }\end{array}$ & $\begin{array}{l}\text { Distorionen } \\
\text { y Adiciones }\end{array}$ & $\begin{array}{l}\text { Erroras } \\
\text { Intrac }\end{array}$ & $\begin{array}{l}\text { Erroves: } \\
\text { Inter. }\end{array}$ & Coner & 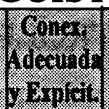 & $\begin{array}{l}\text { Coner } \\
\text { Adec } \\
\text { elinplies }\end{array}$ \\
\hline \multicolumn{10}{|l|}{ Modul Comp: } \\
\hline \multicolumn{10}{|l|}{ Modul. Incom. } \\
\hline Modul Insuf: & $-.735^{* *}$ & & & & & & & & \\
\hline Distors. y Adic & $-.740 * *$ & & $.619 * *$ & & & & & & \\
\hline Error. Intra. & $-.749 * *$ & & $.612 * *$ & $.707 * *$ & & & & & \\
\hline Error. Inter. & $-.483^{*}$ & & $.574 * *$ & & $.580 * *$ & & & & \\
\hline Con. Inadec. & $-.833^{* *}$ & & $.870 * *$ & $.797 * *$ & $.815^{* *}$ & $.491 *$ & & & \\
\hline Contadec.Exp. & $.676 * *$ & & $-.597 * *$ & $-579 * *$ & $-.539 *$ & & $-.687 * *$ & & \\
\hline Con.Adec.Imp & $.558 * *$ & & $-.678 * *$ & $-.593 * *$ & $-.652 * *$ & & $-.778 * *$ & & \\
\hline No Cons.Text: & $.523 * *$ & & $-.672 * *$ & $-.697 * *$ & $-.611 * *$ & & $-.821 * *$ & $\mid .518 *$ & $679 * *$ \\
\hline
\end{tabular}

$* *$ La correlación es significativa al nivel 0,01 (bilateral)

$*$ La correlación es significativa al nivel 0,05 (bilateral)

TABLA N ${ }^{\circ}$ 5: COMPARACIÓN DE LOS ÍND. DE LAS DIMENSIONES QUE COMPONEN LA MACROESTRUCTURA DEL TEXTO ENTRE SUJETOS CON ESQUIZ. Y CONTROLES (T0)

\begin{tabular}{|c|c|c|c|c|c|c|}
\hline ÍNDICES & GRUPO & $\mathrm{N}$ & Media & $\mathrm{dt}$ & $\mathrm{z}$ & Sig. (bilat) \\
\hline Modulos comp. & Ss con esquizofrenia & 10 & 12.5 & 27.00 & 2.53 & .011 \\
& Ss controles normales & 11 & 52.7 & 35.08 & & \\
\hline Modulos incomp. & Ss con esquizofrenia & 10 & 31.6 & 29.5 & 2.14 & .831 \\
& Ss controles normales & 11 & 34.6 & 25.8 & & \\
\hline Modulos insuf: & Ss con esquizofrenia & 10 & 55.9 & 41.2 & 2.42 & .016 \\
& Ss controles normales & 11 & 12.7 & 15.8 & & \\
\hline Distors. y.adic. & Ss con esquizofrenia & 10 & 67.0 & 20.96 & 3.40 & .001 \\
\hline Ss controles normales & 11 & 25.3 & 19.39 & & \\
\hline Errores intra- & Ss con esquizofrenia & 10 & 46.9 & 24.6 & 2.77 & .006 \\
\hline conistituyentes & Ss controles normales & 11 & 16.2 & 15.1 & & \\
\hline Errores inter- & Ss con esquizofrenia & 10 & 23.7 & 27.6 & 2.91 & .004 \\
\hline constituyentes & Ss controles normales & 11 & .00 & .00 & & \\
\hline Conex. inadec. & Ss con esquizofrenia & 10 & 63.6 & 35.19 & 2.95 & .003 \\
\hline Conex: adec: & Ss controles normales & 11 & 13.9 & 12.41 & & \\
\hline Ss con esquizofrenia & 10 & 28.02 & 34.12 & 2.52 & .012 \\
\hline Conplicitas. & Ss controles normales & 11 & 70.65 & 21.34 & & \\
\hline e implicitas & Ss con esquizofrenia & 10 & 8.38 & 11.93 & 1.12 & .263 \\
\hline No de constit. & Ss con esquizofrenia & 10 & 85.0 & 19.36 & 1.72 & .080 \\
& Ss controles normales & 11 & 97.7 & 5.05 & & \\
\hline
\end{tabular}


TABLA N ${ }^{\circ}$ 6: COMPARACIÓN DE LOS ÍNDICES DE LAS DIMENSIONES QUE COMPONEN LA MACROESTRUCTURA DEL TEXTO ENTRE SUJETOS CON ESQUIZOFRENIA Y CONTROLES (T1)

\begin{tabular}{|c|c|c|c|c|c|c|}
\hline indict & - GRUPO & $\mathbf{N}$ & Media & dit. & 2 & Sig. (bilat) \\
\hline Módulos completos & $\begin{array}{l}\text { Ss con esquizofrenia } \\
\text { Ss controles normales }\end{array}$ & $\begin{array}{l}10 \\
11\end{array}$ & $\begin{array}{l}13.7 \\
46.6\end{array}$ & $\begin{array}{l}16.0 \\
24.4\end{array}$ & 2.83 & .005 \\
\hline Mod. incompletos & $\begin{array}{l}\text { Ss con esquizofrenia } \\
\text { Ss controles normales }\end{array}$ & $\begin{array}{l}10 \\
11\end{array}$ & $\begin{array}{l}37.7 \\
38.6\end{array}$ & $\begin{array}{l}26.2 \\
17.2\end{array}$ & .288 & .774 \\
\hline Mod. insunficientes & $\begin{array}{l}\text { Ss con esquizofrenia } \\
\text { Ss controles normales }\end{array}$ & $\begin{array}{l}10 \\
11\end{array}$ & $\begin{array}{l}48.6 \\
14.8\end{array}$ & $\begin{array}{l}37.3 \\
16.0\end{array}$ & 2.62 & .024 \\
\hline istor vadicion & $\begin{array}{l}\text { Ss con esquizofrenia } \\
\text { Ss controles normales }\end{array}$ & $\begin{array}{l}10 \\
11 \\
\end{array}$ & $\begin{array}{l}66.4 \\
15.9 \\
\end{array}$ & $\begin{array}{l}66.4 \\
15.9\end{array}$ & 3.48 & .000 \\
\hline $\begin{array}{l}\text { Errores intra- } \\
\text { constituyentes }\end{array}$ & $\begin{array}{l}\text { Ss con esquizofrenia } \\
\text { Ss controles normales }\end{array}$ & $\begin{array}{l}10 \\
11\end{array}$ & $\begin{array}{l}57.1 \\
12.5\end{array}$ & $\begin{array}{l}29.4 \\
15.8\end{array}$ & 3.23 & .001 \\
\hline $\begin{array}{l}\text { Errores inter- } \\
\text { constituyentes }\end{array}$ & $\begin{array}{l}\text { Ss con esquizofrenia } \\
\text { Ss controles normales }\end{array}$ & $\begin{array}{l}10 \\
11\end{array}$ & $\begin{array}{l}16.6 \\
0.00\end{array}$ & $\begin{array}{l}23.8 \\
0.00\end{array}$ & 1.81 & .071 \\
\hline $\begin{array}{l}\text { Conexiones } \\
\text { Inadecuadas }\end{array}$ & $\begin{array}{l}\text { Ss con esquizofrenia } \\
\text { Ss controles normales }\end{array}$ & $\begin{array}{l}10 \\
11\end{array}$ & $\begin{array}{l}58.6 \\
11.7\end{array}$ & $\begin{array}{l}35.3 \\
12.5\end{array}$ & 3.14 & .002 \\
\hline $\begin{array}{l}\text { Coner adect } \\
\text { y explicitad }\end{array}$ & $\begin{array}{l}\text { Ss con esquizofrenia } \\
\text { Ss controles normales }\end{array}$ & $\begin{array}{l}10 \\
11\end{array}$ & $\begin{array}{l}14.3 \\
36.4\end{array}$ & $\begin{array}{l}13.5 \\
23.3\end{array}$ & 2.40 & .016 \\
\hline $\begin{array}{l}\text { Comer adects } \\
\text { e implicitas }\end{array}$ & $\begin{array}{l}\text { Ss con esquizofrenia } \\
\text { Ss controles normales }\end{array}$ & $\begin{array}{l}10 \\
11 \\
\end{array}$ & $\begin{array}{l}27.1 \\
51.9\end{array}$ & $\begin{array}{l}24.7 \\
20.5\end{array}$ & 1.98 & .047 \\
\hline $\mathrm{N}^{0} \mathrm{de}$ constit. & $\begin{array}{l}\text { Ss con esquizofrenia } \\
\text { Ss controles normales }\end{array}$ & $\begin{array}{l}10 \\
11\end{array}$ & $\begin{array}{l}81.3 \\
100\end{array}$ & $\begin{array}{l}28.4 \\
0.00\end{array}$ & 2.16 & .030 \\
\hline
\end{tabular}


TABLA N ${ }^{\circ}$ 7: COMPARACIÓN DE LOS ÍNDICES DE LAS DIMENSIONES QUE COMPONEN LA MACROESTRUCTURA DEL TEXTO ENTRE LOS SUJETOS CON ESQUIZOFRENIA (T0 y T1)

\begin{tabular}{|c|c|c|c|c|c|}
\hline 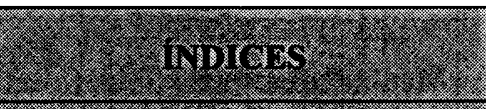 & 13 & Mealn & $\mathrm{dt}$ & $(2,8$ & Sig (bin). \\
\hline 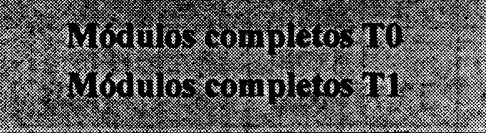 & 10 & $\begin{array}{l}12.5 \\
13.7\end{array}$ & $\begin{array}{l}27.0 \\
16.1\end{array}$ & .632 & .901 \\
\hline 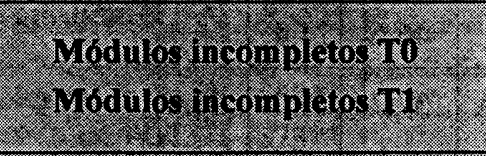 & 10 & $\begin{array}{l}31.6 \\
37.7\end{array}$ & $\begin{array}{l}29.5 \\
26.1\end{array}$ & .281 & .519 \\
\hline $\begin{array}{l}\text { Modulos insuricientes To } \\
\text { Modulos insuricientes } 171\end{array}$ & 10 & $\begin{array}{l}55.9 \\
48.8\end{array}$ & $\begin{array}{l}41.2 \\
37.3\end{array}$ & .771 & .646 \\
\hline $\begin{array}{l}\text { Distorsiones y adiciones T0 } \\
\text { Distorsioties y adiciones Th }\end{array}$ & 10 & $\begin{array}{l}67.0 \\
66.4\end{array}$ & $\begin{array}{l}20.9 \\
29.1\end{array}$ & 297 & .958 \\
\hline $\begin{array}{l}\text { Errores intraconstituyentes TO } \\
\text { Errores intraconstituyentes TI }\end{array}$ & 10 & $\begin{array}{l}46.9 \\
57.4\end{array}$ & $\begin{array}{l}24.6 \\
29.4\end{array}$ & 1.14 & .256 \\
\hline $\begin{array}{l}\text { Errores interconstituyentes To } \\
\text { Errores intercoonstituyentes T1 }\end{array}$ & 10 & $\begin{array}{l}23.7 \\
16.6\end{array}$ & $\begin{array}{l}27.6 \\
23.8\end{array}$ & 1.16 & 248 \\
\hline $\begin{array}{l}\text { Concxiones findecuadas } \mathrm{TO}^{-} \\
\text {Conexiones inadecuadas TI }\end{array}$ & 10 & $\begin{array}{l}63.6 \\
58.6\end{array}$ & $\begin{array}{l}35.2 \\
35.3\end{array}$ & .561 & .730 \\
\hline $\begin{array}{l}\text { Coneriones adec y erpl.To } \\
\text { Conteriones adec. y expl. } 171\end{array}$ & 10 & $\begin{array}{l}28.0 \\
14.3\end{array}$ & $\begin{array}{l}34.1 \\
13.4\end{array}$ & .845 & .257. \\
\hline $\begin{array}{l}\text { Coneriones adec. e impl. To } \\
\text { Conexiones adec. eimpl.T1 }\end{array}$ & 10 & $\begin{array}{l}8.38 \\
27.1\end{array}$ & $\begin{array}{l}11.9 \\
24.7\end{array}$ & 2.37 & .008 \\
\hline $\begin{array}{l}\text { Número de constituyentes } \mathrm{TO} \\
\text { Número de constituyentes } \mathrm{T} 1\end{array}$ & 10 & $\begin{array}{l}85.0 \\
81.2\end{array}$ & $\begin{array}{l}19.4 \\
28.4\end{array}$ & .425 & .591 \\
\hline
\end{tabular}



ORIGINALES Y REVISIONES

TABLA No 8: COMPARACIÓN DE LOS ÍNDICES DE LAS DIMENSIONES QUE COMPONEN LA MACROESTRUCTURA DEL TEXTO ENTRE LOS SUJETOS CONTROLES NORMALES (T0 y T1)

\begin{tabular}{|c|c|c|c|c|c|}
\hline 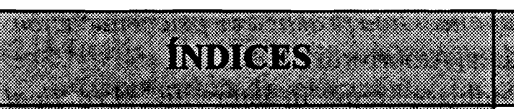 & 4. & Media & dt & $\sqrt{12}$ & Sig. (bilat.) \\
\hline $\begin{array}{l}\text { Modulos completos To } \\
\text { Modulos completos TI }\end{array}$ & 11 & $\begin{array}{l}52.8 \\
46.6\end{array}$ & $\begin{array}{l}35.0 \\
24.4\end{array}$ & .845 & .398 \\
\hline $\begin{array}{l}\text { inodiuos medimpletos To } \\
\text { Modulos incompletos. }\end{array}$ & 11 & $\begin{array}{l}34.6 \\
38.6\end{array}$ & $\begin{array}{l}25.8 \\
17.2\end{array}$ & .582 & .560 \\
\hline $\begin{array}{l}\text { Modulos insuncientes mo } \\
\text { Mogtios insuriegrantes T1 }\end{array}$ & 11 & $\begin{array}{l}12.7 \\
14.8\end{array}$ & $\begin{array}{l}15.8 \\
16.6\end{array}$ & .755 & .450 \\
\hline 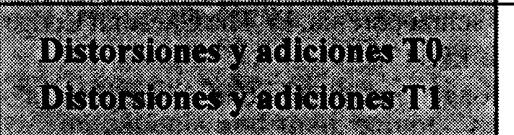 & 11 & $\begin{array}{l}25.3 \\
15.9\end{array}$ & $\begin{array}{l}19.4 \\
14.9\end{array}$ & 1.63 & 103 \\
\hline 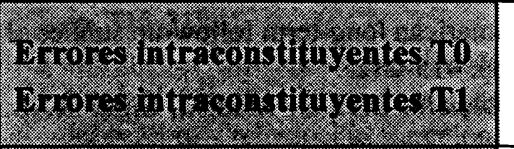 & 11 & $\begin{array}{l}16.2 \\
12.5\end{array}$ & $\begin{array}{l}15.1 \\
15.8\end{array}$ & .850 & .395 \\
\hline 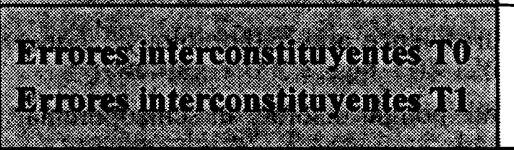 & 11 & $\begin{array}{c}0^{*} \\
2.27\end{array}$ & $\begin{array}{c}0 \\
5.05\end{array}$ & 1.41 & . 157. \\
\hline 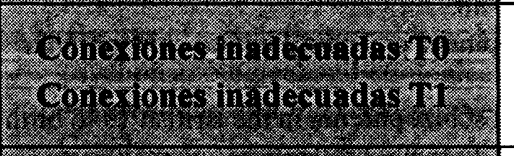 & 11 & $\begin{array}{l}15.2 \\
11.7\end{array}$ & $\begin{array}{l}14.6 \\
12.5\end{array}$ & .635 & .526 \\
\hline 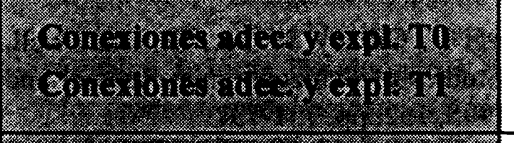 & 11 & $\begin{array}{l}69.4 \\
36.7\end{array}$ & $\begin{array}{l}24.4 \\
23.3\end{array}$ & 2.58 & .010 \\
\hline 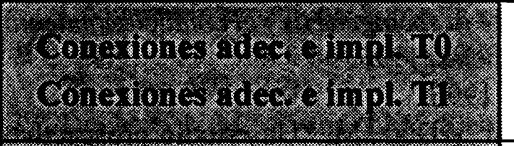 & 11 & $\begin{array}{l}15.4 \\
51.9\end{array}$ & $\begin{array}{l}16.3 \\
20.4\end{array}$ & 2.80 & .005 \\
\hline 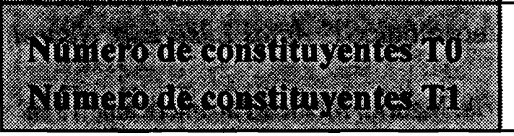 & 11 & $\begin{array}{l}97.7 \\
100\end{array}$ & $\begin{array}{c}5.05 \\
0\end{array}$ & 1.41 & .157 \\
\hline
\end{tabular}

* No se puede calcular la t porque el error estándar de la diferencia es 0. 


\section{5.- BIBLIOGRAFÍA}

(1) Strauss, J.S. y Carpenter W.T. "The prediction of recovery in schizophrenia III. Five year outcome an its predictors". Arch Gen Psychatry, 1977, 34, pp. 159-163.

(2) Anderson C.; Reiss D.; y Hogarty G. Esquizofrenia y familia. Guia práctica de psicoeducación. Guilford Press. (Traducción Valcárcel Z.J., Buenos Aires, Amorrortu), 1986.

(3) Cohen, I. "Studies of the course and outcome of schizophrenia in later life". Practical Geriatrics,1995, 46, 9, pp. 877-889.

(4) Brandley P.B. y Hirsch S.R. "The Psychopharmacology and treatment of schizophrenia". Oxford Medical Publication, 1986, pp. 27-709.

(5) Bleuler, M. "A 23 years longitudinal study of 208 schizophrenics impressions in regard to the nature of schizophrenia". En: D. Rosenthal y S.S. Kety (Ed.). The transmission of schizophrenia. New York. Pergamon Press, 1968.

(6) World Health Organization. The International pilot study of schizophrenia. Geneve W.H.O., 1973

(7) Davis J.M. y Mostert A.M. "Biological treatment of schizophrenia disorders". En: S. Arieti (Ed.). American Handbook of Psychiatry, Vol. 8, Biological Psychiatry Basic Books, New York., 1986.

(8) McGlashan, T.H. "The prediction of outcome in chronic schizophrenia. IV The Chestnut Lodge Follow-up Study”. Arch. Gen. Psychiatry, 1986, 43, pp. 157-176.

(9) McGlashan, T.H. "Predictors of shorter, medium and longe term ouctome in schizophrenia". American Journal of Psychiatry, 1986, 143, 1, pp. 50-55.

(10) McGlashan, T.H. "A selective review of recent North American long-term follow-up studies of schizophrenia". Schizophrenia Bulletin, 1988, 14, 4, pp. 415-442.

(11) Colodrón, A. Las esquizofrenias. Síndrome de Kraepelin-Bleuler. Madrid, Siglo XXI., 1990

(12) Waddington J.L. "Schizophrenia: Developmental neuroscience and pathobiology". Lancet 1993, 34, pp. 531-536.

(13) Barch, D. y Berembaum, H. "Language production and thought disorder in schizophrenia". Journal of Abnormal Psychology, 1996, 105, 1 pp., 81-88.

(14) Jones, P., Murray, R.M. y Rodgers, B. "Child development preceding adult schizophrenia". Schizophrenia Research, 1994, 11,pp. 97.

(15) Jones, P. et al. "Child development risk factors for adult schizophrenia in the British 1946 birth cohort”. Lancet, 1994, 344, pp. 1398-1402.

(16) Arnold, S.; Gur, R.; Shapiro, R.; Fisher, K.; Moherg, P.; Gibney, M.Gur, R.; Blackwell, P. y Trojanowski, J. "Prospective Clinicopathologic Studies of Schizophrenia: Accrual and Assessment of Patients". Am. Journal of Psychiatry, 1995, 152, pp. 731-737

(17) Gutierrez, M.; Ezcurra, J.y Pichot, P. Esquizofrenia. Entre la sociogénesis y el condicionamiento biológico I, II y III. Barcelona, Ediciones en Neurociencias, 1995.

(18) Chinchilla, A. Las esquizofrenias. Barcelona, Masson, 1996.

(19) Chinchilla, A.; Vega, M.; Cebollada, A.; Montes, J.M. y Diaz, M. "Un estudio prospectivo de pacientes con trastorno esquizofreniforme: ¿dilema nosológico?” Actas Luso-Esp. Neurol. Psiquiatr., 1996, 24, 5, pp. 245-251.

(20) Kaplan, H.I.; Sadock, B.J. y Grebb, J.A. Sinopsis de Psiquiatria. Madrid, Médico-Panamericana, 1996.

(21) Sáiz Ruiz, J. Esquizofrenia: Enfermedad del cerebro y reto social. Barcelona, Masson, 1999. 
(22) Alaghband-Rad, J. McKenna, K; Gordon, C.; Albus, K. et al. "Chilhood-onset schizophrenia: The severity of premorbid course". Journal of the Academy of Child and Adolescent Psychiatry, 1995, Vol. 34, 10, pp. 1273-1283

(23) Colodrón, A. El trastorno esquizofrénico de la acción humana. Madrid, Fundación Archivos de Neurobiología, 1995.

(24) Hollis, C. "Child and adolescent (juvenile onset) schizophrenia. A case control study of premorbid developmental impairments". British Journal of Psychiatry, 1995, 166, 4, pp. 489-495.

(25) Wieselgren I.M., Lindström L.H. "A prospective 1-5 year outcome study in first admitted and readmitted schizophrenic patients; relationship to heredity, premorbid adjustment, duration of disease and education level at index admission and neuroleptic treatment". Acta Psychiatr. Scand. 1996, 93, pp. 9-19.

(26) Kay, S.R. y Lindenmayer, J.P. "Outcome predictors in acute schizophrenia. Prospective significance of background and clinical dimensions". Journal of Nervous and Mental Diseases, 1987, 175, 3, pp. 152-160.

(27) Quinlan, D.M.; Schuldberg, D.; Morgenstern, H. y Glazer, W. "Positive and Negative Symptom Course in Chronic Community-Based Patients: A Two-Year Prospective Study”. British Journal of Psychiatry, 1996, 169, pp. 580-586.

(28) Goldstein, M. J. "Preventive intervention in shizophrenia ; are we ready?",en DHHS publication, 82-111.1982- Government printing office, Washington DC.

(29) Docherty, N.M.; Sledge, W.H.; Wexler, B.E. "Affective reactivity in stable schizophrenic outpatients and their parents". Journal of Nervous and Mental Disease, 1994, 182, 6, pp. 313-318.

(30) Martinez, P.C.; Lemos, S y Bobes, J. "Indicadores cognitivos en los trastornos del espectro esquizofrénico: en busca de nuevos marcadores”. En J. Sáiz (Dir.): Esquizofrenia: Enfermedad del cerebro y reto social. Barcelona, Masson. 1999.

(31) Zubin, J. y Spring, B. "Vulnerability: A new view of schizophrenia". Journal of Abnormal Psychology, 1977, 86, pp. 103-126.

(32) Zubin, J., Magaziner, J. y Steinhauer, S. "The metamorphosis of schizophrenia: from chronicity to vulnerability”. Psychological Medicine, 1983, 13, pp. 551-571.

(33) Parnas, J.; Schulsinger, F. y Mednick, A. "The Copenhagen high-risk study: major psychopathological and etiological findings”. En: E. Straube y K. Hahlweg (Eds.). Concepts, Vulnerability and Intervention. New York, Springer-Verlag, 1990.

(34) Grijalvo, J. "Evolución clínica de distintos subtipos de esquizofrenia con especial referencia al lenguaje". Tesis Doctoral no publicada, 1991.

(35) Grijalvo, J.; Insúa, P. y Guimón, J. Evolución y potencial predictivo de la sintomatología clínica, los procesos psicolingüisticos y los subtipos de esquizofrenia. Comunicación presentada en el Congreso Internacional sobre "Las Psicosis", Palencia, abril de 1997.

(36) Alpert, M. "The signs and symtoms of schizophrenia". Comprehensive Psychiatry, 1985, 26, 2, pp. 103-112.

(37) Docherty,N.M.; Evans, I.M.; Sledge, W.H.; Seibyl, J.P. y Krystal, J.H. “Affective reactivity of language in schizophrenia". Journal of Nervous and Mental Disease, 1994, 182, 2,pp. 98-102.

(38) Condray, R., Steinhauer, S.R.; Van Kammen, D.P.; y Kasparek, A. "Working memory capacity predicts language comprehension in schizophreniac patients". Schizophrenia Research, 1996, 20, 1-2, pp. 1-13. 
(39) Lieberman J.A. "Signs and Symptoms; What Can They Teil Us About the Clinical Course and Pathophysiologic Processes of Schizophrenia?" Arch Gen Psychiatry, 1995, vol 52, pp. 361-363.

(40) Crow, T.J.; Done, D.J. y Sacker, A. "Chilhood precursors of psychosis as clues to its evolutionary origins". European Archives of Psychiatry, 1995, 245, 2, pp. 61-69.

(41) Opler, L.A., Kay S.R. y Fiszbein A. "Positive and negative Syndromes in schizophrenia: Typological, dimensional, and pharma cological validation". En: Harvey y Walker (Eds.). Positive and negative symptoms of psychosis. Hillsdal N.J.: L.E.A. 1987.

(42) Oltmanns, T.F., Murphy, R., Berembaum, H. y Dunlop, S.R. "Rating verbal communication impairment in schizophrenia and affective disorders". Schizophrenia Bulletin, 1985, 11, 2; pp. 292-299.

(43) Silverstein, M.; Harrow, M. y Marengo, J. "Disordered thinking and cerebral dysfunction: Laterality effects, language, and intellectual functions". Archives of Clinical Neuropsychology, 1993, 8, 6, pp. 497-509.

(44) Barch, D. y Berembaum, H. "The relationship between information processing and language production”. Journal of Abnormal Psychology, 1994, 103, 2,pp. 241-250.

(45) Taylor, M.A.; Reed, R. y Berembaum, S. "Paterns of Speech in Schizophrenia and Mania". Journal of Nervous and Mental Diseases, 1994, 182, pp. 319-326.

(46) Andreasen, N.C., Tsuang, M.T. Y Canter, A. "The significance of thought disorder in diagnostic evaluations”. Comprehensive Psychiatry, 1974, 15, pp. 27-34.

(47) Harrow, M.; Silverstein, M. y Marengo, J. “Disordered Thinking : Does it identify nuclear Schizophrenia?”'Arch. Gen. Psychiatry, 1983, 40, pp. 765-771.

(48) Grijalvo, J. y Guimón, J. Predictores lingüisticos de la evolución de la Esquizofrenia. XIX Congreso Nacional de la Sociedad Española de Psiquiatría; Edit. ELA, 1994, pp. 11-29.

(49) Andreasen, N.C. "Thought, language and communication disorders. Clinical assessment, definition of terms and evaluation of their reliability". Archives of General Psychiatry, 1979, 36, pp. 1315-1323.

(50) Andreasen, N.C. "Thought, language and communication disorders. Diagnostic significance". Archives of General Psychiatry,1979, 36, pp. 1325-1330.

(51) Miller -Del. D; Arndt, S. y Andreasen, N. "Alogia, attentional impairement, and inappropiate affect: Their status in the dimensions of schizophrenia". Comprehensive Psychiatry,1993, 34,4 , pp. 221-226.

(52) Knight, R.A., Elliot, D.S., Roff, J.D. y Watson, C.G. "Concurrent and predictive validity of components of disordered thinking in schizophrenia", Schizophrenia Bulletin, 1986, 12, pp. 427-446.

(53) Ragin, A. y Oltmanns, T.F. "Communicability and thought disorder in schizophrenics and other diagnostic groups: A follow-up study". British Journal of Psychiatry, 1987, 150, pp. 494-500.

(54) Thomas, P.; King, K. y Fraser W.I. "Positive and Negative Symptoms of Schizophrenia and Linguistic performance”. Acta Psychiatr. Scand, 1987, 76; pp. 144-151.

(55) Barrelet, L.; Piguet, D. y Corradini; Corradini, S. "Schizophrenie et troubles du langage". Encephale, 1993, 19, 5, pp. 533-540.

(56) Anand, A.; Wales, R.J.; Jackson, H.J. y Copolov, D.L. "Linguistic impairment in early psychosis". Journal of Nervous and Mental Disease, 1994, 182, 9, pp. 488-493.

(57) Baltaxe, C.A. y Simmons, J.Q. "Speech and language disorder in children and adolescents with schizophrenia”. Schizophrenia Bulletin,1995, 21, 4, pp. 677-692. 
(58) Andreasen, N.C. y Grove, W.M. "The relationship between schizophrenic language manic languaje and aphasia”. En J. Gruzelier y P. Flor-Henry (Eds): Hemisphere asymmetries and function in psycopathology. Elsevier: North Holland Press; 1979, pp. 373-390.

(59) Grove, W.M., Andreasen, N.C. "Language and thinking in psychosis. Is there an input abnormality?" Arch. Gen. Psychiatry, 1985, 42, pp. 26-32.

(60) Andreasen, N.C. "Positive vs negative schizophrenia: a critical evaluation". Schizophrenia. Bulletin, 1985, 11, 3, pp. 380.

(61) Andreasen, N.C. y Olsen, S. "Negative vs Positive schizophrenia: Definition and validation". Arch. Gen. Psychiatry, 1982, 39; pp. 789-794.

(62) Harrow, M. y Quinlan, D.M. Disorderer thinking and schizophrenic psychopathology. New York, Garden Press Inc., 1985.

(63) Marengo, J. y Harrow, M. "Thought disorder: A function of schizophrenia, mania or psychosis". Journal of Nervous and Mental Disease, 1985, 173,pp. 35-41.

(64) Belinchón, M. Producción verbal y esquizofrenia: Una aproximación psicolingüística al estudio de las alteraciones del discurso. Tesis Doctoral no publicada. 1986.

(65) Belinchón, M. “Lenguage”. En J.M. Ruiz-Vargas (Dir.).: Esquizofrenia: Un enfoque cognitivo. Madrid, Alianza., 1987

(66) Belinchón, M. "Hacia una caracterización empírica del lenguaje esquizofrénico". Estudios de Psicologia, 1988, 33-34, pp. 157-189.

(67) Belinchón, M. “Comunicación y lenguaje en la esquizofrenia”. En: C. Castilla del Pino y J.M. Ruiz Vargas (Eds.). Aspectos cognitivos de la esquizofrenia. Madrid, Trotta, 1991.

(68) Mantell, J.; DiVittis, A. y Auerbach, M. Evaluating Prevention Interventions. NY, Plenum Press, 1997.

(69) Obiols, JE.; Salvador, L.; Humbert, M. y Obiols, J. "Evaluación de los síntomas negativos en la esquizofrenia". Revista del Depto. de Psiquiatría de la Fac. de Medicina de Barcelona, 1985,12 , pp. 85-91.

(70) Peralta, V.; Cuesta, M.J. y De León, J. "Estudio de la fiabilidad inter-observador de los síntomas negativos esquizofrénicos”. Actas Luso-Esp Neurol Psiquiatr, 1991, 19, pp.221-225.

(71) Peralta, V.; De León, J. y Cuesta, M.J. "Are there more than two syndromes in schizophrenia? A critique of the positive-negative dicotomy". Br. J. Psichiatr, 1992, 161, pp.335-343.

(72) Vazquez Barquero, J.L.; Lastra, Y.; Cuesta, M.J.; Herrera, S. y Dunn. "Patterns of positive and negative symptoms in first episode schizophrenia". British Journal of Psychiatry, 1996, 168: pp. 693-701.

(73) Andreasen, N.C. "Thought, Language and Communication disorders. Clinical assessment, definition of terms and evaluation of their reliability". Archives of General Psychiatry, 1979, 36, pp. 1315-1321.

(74) Obiols, JE.; Salvador, L.; Humbert, M. y Obiols, J. Escala para la evaluación del Pensamiento, Lenguaje y Comunicación, 1985, Universidad de Barcelona.

(75) Peralta, V.; Cuesta, M.J. y De León, J. "Formal Thought Disorder in Schizophrenia: a factor analytic study". Comprehensive Psychiatric, 1992, 33, pp. 105-110.

(76) Endicott, J.; Spitzer, R.L.; Fleiss, J.L. y Cohen, J. "The global assessment scale-A procedure for measuring overall severity of psychiatric disturbance". Arch. Gen Psychiatry, 1976, 33, pp. 766-771

(77) Spitzer, R.L., Andreasen, N. y Endicott, J. "Schizophrenia and other psychotic disorders in DSM-III", Schizophrenia Bulletin, 1978, 4, pp. 489-509.

(78) Strauss, J.S. y Carpenter W.T. "The prediction of outcome in schizophrenia: relationships between predictor and outcome variables”. Arch. Gen. Psychiatry. 1984, 31, pp. 37-42. 
(79) Ruiz M.A., Baca E. "Cuestionario de Calidad de Vida". En: J. Bobes, MP. Gonzalez, M. Bousoño (Eds.). Calidad de vida en las esquizofrenias. Madrid: JR Prous, 1995.

(81) Canon-Spoor, H.E.; Potkin, S.G. y Wyatt, R.J. "Measurement of premorbid adjustement in Chronic schizophrenia". Schizophrenia Bulletin, 1982, 8, pp. 470-484.

(82) Overall, J.E.y Gorham, D.R. The Brief Psychiatric Scale. Psychological Reports, 1962, 10 pp. 799-812.

(83) De las Cuevas, C., et al. "Análisis factorial de la versión española del SCL-90-R en la población general". Anales de Psiquiatría, 1991, 7, pp. 93-96.

(84) Van Dijk, T.A. Texto y Contexto. Madrid, Cátedra, 1980.

(85) Van Dijk, T.A. "Recalling and sumarizing complex discourse". En W. Burghardt y.K. Hölker (Ed.) Text processing. Berlin, Walter de Gruyter, 1979.

(86) Ruiz Vargas, J.M. Esquizofrenia. Un enfoque cognitivo. Barcelona, Paidós, 1987.

(87) Ruiz Vargas, J..M. "Psicología cognitiva y esquizofrenia: Una aproximación teórica". En C. Castilla del Pino y J.M. Ruiz Vargas (Eds.). Aspectos cognitivos de la esquizofrenia. Madrid, Trotta, 1991

*Dra. en Psicología. Profesora del Depto. de Psicología Básica de la Universidad del País Vasco

**Dr. en Medicina. Psiquiatra. Osakidetza/Servicio Vasco de Salud

***Dr. en Psicología. Profesor del Depto. de Psicología Básica de la Universidad del País Vasco

Correspondencia: Dra. Patricia Insúa - Facultad de Psicología - Av. de Tolosa, 70 20018 San Sebastián - Tel.: 943-015735 Fax: 943-311055 - e-mail: pbpincep@sc.ehu.es

Fecha de recepción: 4-12-00 